Oikos 120: 1826-1837, 2011

doi: $10.1111 / j .1600-0706.2011 .19438 . x$

(C) 2011 The Authors. Oikos (C) 2011 Nordic Society Oikos

Subject Editor: Tim Benton. Accepted 5 September 2011

\title{
Directions in modelling partial migration: how adaptation can cause a population decline and why the rules of territory acquisition matter
}

\author{
Hanna Kokko \\ H. Kokko(hanna.kokko@anu.edu.au), Evolution, Ecology and Genetics, Research School of Biology, The Australian National Univ., Canberra, \\ ACT 0200, Australia.
}

\begin{abstract}
Modelling of partial migration in birds has progressed from simple graphical representations to sophisticated analyses that use evolutionary invasion analysis to determine how the success of the two strategies (stay year round on the breeding grounds, or migrate) can become frequency dependent. Here I build two models to relax two assumptions commonly made in models and often violated in nature: that individuals do not vary in any trait other than their migratory propensity, and that the prior residence effect (which grants priority access of good habitats to non-migrants) operates at maximum strength. The same framework can incorporate and merge aspects of various hypotheses proposed to explain partial migration (dominance, body size, arrival timing, and limited foraging opportunities), and shows that either small (subdominant) or large (dominant) individuals may emerge as the more likely migrants; the latter case occurs when it is easy for socially dominant migrants to win back prime breeding locations upon their arrival. The dynamics of territory acquisition is shown to be an important and understudied topic, as variations in the relative importance of prior residency versus resource holding power can shift a population from complete migration to complete year-round residency. These models also highlight exceptions to a tacit assumption in discussions of evolution of migration under climate change, which is that populations can decline if genetic adaptation or phenotypic plasticity do not occur fast enough. Competition can also yield the opposite pattern where adaptation itself leads to a population decline.
\end{abstract}

Alternative strategies that coexist within a population fascinate evolutionary biologists and ecologists for a good reason (Sinervo and Calsbeek 2006). A proper explanation of discrete morphs or behaviours requires balancing selection, which usually takes the form of frequency-dependent selection of one type or another - including 'best of a bad job' explanations where some individuals are forced to use one option because others have already taken another more profitable one. If there is no frequency dependence of any kind, and no genetic quirk such as a heterozygote advantage operates either, then one of the behaviours should be eventually lost. This can happen through selection if one behavioural type or morph performs consistently less well than another (without it being a 'best of a bad job' which implies that individuals following the strategy will switch to a more profitable option as soon as opportunities arise). Or, if there is no predictable difference between the fitness of types regardless of their relative frequency, then drift is expected to perform the same removal job, albeit more slowly.

Proper examples of frequency-dependence in nature are, however, not very common. Partial migration is a fascinating example where researchers have for a number of decades already commented on reasons why the success of the non-migratory and migratory strategies may depend on the number of individuals performing them (Lundberg 1987, 1988, reviewed by Chapman et al. 2011). Since then, our theoretical understanding of partial migration in birds has progressed from simple graphical representations (Lundberg $1987,1988)$ to examinations that use evolutionary invasion analysis to evaluate the fitness of a rare mutant in an environment 'created' by the currently used strategy in the population (Kaitala et al. 1993, Kokko and Lundberg 2001, Kokko 2007, Griswold et al. 2010). The environmental conditions are created by the population in the sense that the intensity of competition for breeding sites and survival can both depend on how many individuals are present and performing each strategy. For example, if no individual attempts year-round residency, then the first arriving migrant in spring has a much wider choice of breeding habitats available than if most of the population has been overwintering. In the latter case, most territories are already occupied by the time the focal migrant arrives (Kokko 2007; note that despite the language of 'winter' and 'spring' conditions, an analogous argument applies for any type of seasonality leading to foraging limitations of other difficulties of surviving during a part of the seasonal cycle: Boyle and Conway 2007, Boyle 2011). Similarly, survival can depend on the local density of individuals. Since local densities depend on the frequency of migration, such 
density dependence can be the basis of frequency-dependent success of strategies, which in turn helps explain how variation in migratory decisions can persist (Kaitala et al. 1993, Taylor and Norris 2007, Griswold et al. 2010).

An intriguing finding from these theoretical studies is that competition for best breeding sites can make individuals accept much higher mortality risks for their attempts to remain year-round in the breeding areas than they would accept for migration. This can lead to paradoxical findings where population size increases when overwintering becomes harder (Fig. 1). Such findings may appear counterintuitive at first sight but become fully explicable once we remind ourselves of a general principle: within-species competition can make individuals invest in traits that primarily aid in outcompeting conspecifics, with no net benefit for the population or species (Rankin et al. 2007). Staying close to the breeding grounds during winter (or, interpreted more globally, during a time period of limited foraging opportunities, Boyle 2008, Jahn et al. 2010) can become a zero-sum game where an individual attempts to access better habitat ahead of others, and the payoffs of such competition can make individuals risk death to the extent that the population stabilizes at a lower size than if all individuals migrated and 'agreed' to compete over territories only once harsh conditions are over. If the period of harsh conditions becomes more severe, more individuals will migrate, and as this is the safer option, the result is a larger breeding population (Fig. 1).

Models such as the ones described above predict that partial migration should only be found when migration confers a net survival benefit for at least some individuals. If year-round residency was both safer and led to better access to territories, migration would never pay off. These models, however, make simplifying assumptions that are unlikely to be fully met in nature. They either model density dependence in a simplistic way that does not explicitly specify the process of breeding site acquisition (Taylor and Norris 2007, Griswold et al. 2010) or, alternatively, they model territories explicitly but base all predictions on the assumption that the 'prior residence effect' operates at full strength. The prior residence effect is the empirical finding, valid across many taxa (Kokko et al. 2006a), that if two equally strong individuals compete over a territory, the one who arrived first tends to win and maintain its ownership. The effect is at its strongest if the prior owner always wins regardless of its strength or proxies of strength such as body size. This is the assumption that brings about the superior breeding site acquisition ability of year-round residents. In reality, however, it is more likely that the effect operates at less than maximal strength, which implies that other individual properties relating to fighting ability (captured by the term RHP, short for resource holding potential) combine with prior ownership to determine the outcome of fights, in a manner described in Fig. 2 (see also Hardy and Field 1998).

Recent empirical work has pointed out that the rules governing partial migration can change dramatically if it is possible for physically dominant migrants to win back prime breeding locations upon their arrival (Jahn et al. 2010). Previous modelling of partial migration has not paid much attention to the process of territory acquisition, even though from the general literature of fighting over resources we know that takeovers can occur if the challenger (e.g. an arriving migrant) has clearly higher RHP than the owner
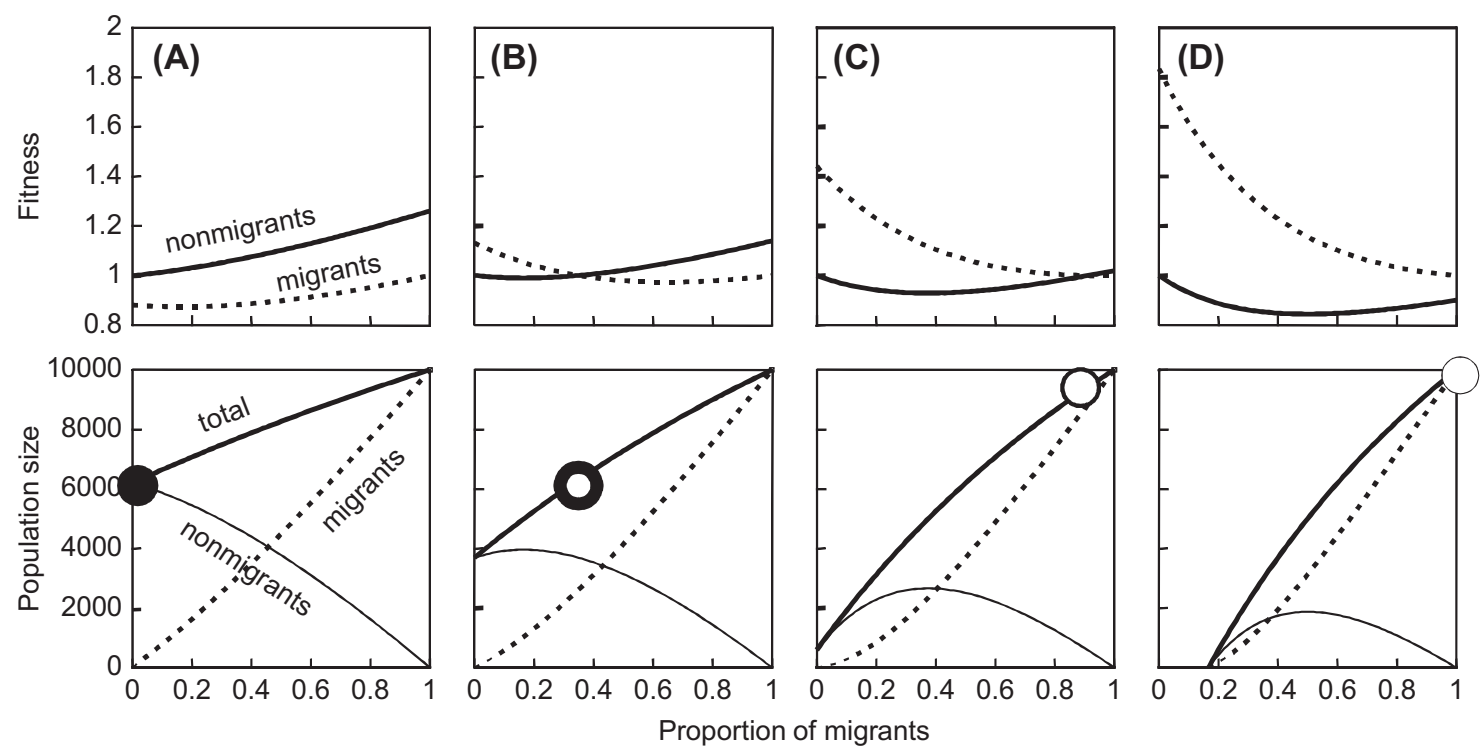

Figure 1. Frequency-dependence and its population consequences in a model with no individual variation other than the migration decision itself. This figure is reproduced from Kokko (2007), but it also becomes the output of Model 1 in the current paper if variance in body size is diminished to zero such that all birds using a specific strategy (migrate or not) have identical survival prospects. Successful overwintering on the breeding grounds becomes increasingly hard from left to right (winter survival for non-migrants is $0.42,0.38,0.34$ and 0.3 respectively, while survival for migrants remains unchanged at 0.5 ). Habitat parameters: $a=2, b=0.0002$ (see Model 1 for explanation). Frequency-dependence diminishes the success of migrants as migration becomes more common (upper row). The amount of white in the large dots of the lower row indicates the proportion of migrants at equilibrium. The total population size increases markedly when more birds choose the safer non-migration option (from left to right); this is interesting because it is a response to deterioration of the environment when attempting year-round residency, not an improvement in the habitat experienced by migrants. 


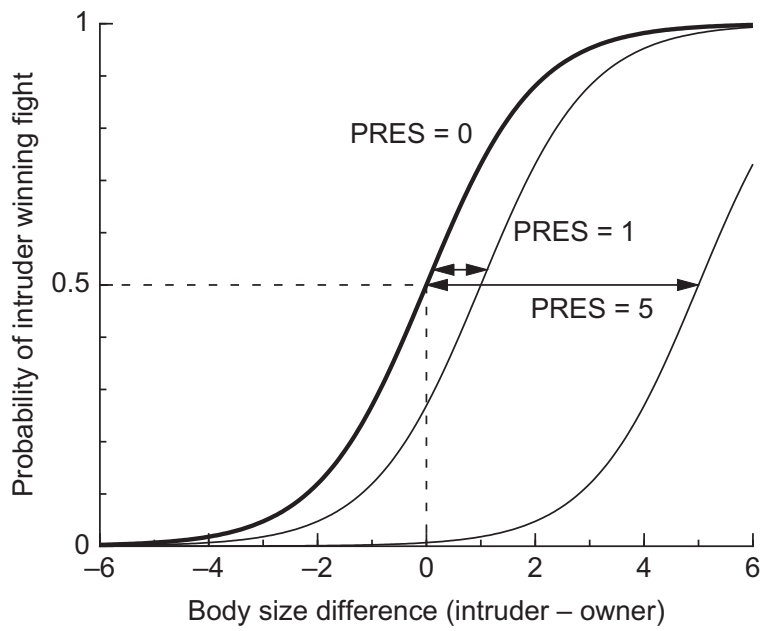

Figure 2. The prior residence effect, illustrated by an example where heaviest birds are $6 \mathrm{~g}$ heavier than the lightest birds. When the effect does not operate (bold curve: prior residence effect strength PRES =0), larger individuals are more likely to win fights regardless of ownership: more than $50 \%$ of fights are won by the intruder as soon as the intruder is heavier than the owner. An effect of strength 1 implies that this curve is shifted by $1 \mathrm{~g}$ to the right (middle curve), thus the intruder has to be $1 \mathrm{~g}$ heavier than the owner to have $50 \%$ probability of winning the fight over ownership. An effect of strength 5 means that intruders very rarely win fights (rightmost curve), and if they do so, they are likely to be very much heavier than the owner. The probability of winning a fight is given as $\left(1+e^{-\left(s_{\mathrm{I}}-\mathrm{s}_{\mathrm{O}}-\mathrm{PRES}\right)}\right)^{-1}$ where $s_{\mathrm{I}}$ is the weight of the intruder, $s_{\mathrm{O}}$ of the owner, and PRES is the prior residence effect strength.

(e.g. an overwintered bird) (reviewed by Kokko et al. 2006a). There are actually two components to this omission from the theoretical literature. One is that individuals are not always equal but can differ in their body size or other traits that can influence not only their RHP but also their ability to survive either migration or non-migration. The other is that takeovers can occur and migrants consequently do not always have to make do with poorer territories than survived nonmigrants (especially if migrants have superior RHP).

Filling in both gaps appears important when understanding condition-dependent (facultative) migratory decisions. Regarding the former, there is plenty of evidence that migrants and non-migrants can differ, and intriguingly, migrants are sometimes less (Smith and Nilsson 1987) and sometimes more dominant (Jahn et al. 2010, see also Boyle 2008) than non-migrants. Regarding the latter, prior residency is certainly known to play a role in migratory species (Sandell and Smith 1991) but simultaneously territorial systems probably rarely operate without takeovers ever occurring. Such takeovers have been argued to explain why dominant individuals in particular can 'have their cake and eat it too', as in tropical kingbirds Tyrannus melancholicus where dominant males survive better as migrants and can then come back to usurp territories from non-migrants (Jahn et al. 2010). This obviously begs the question why any bird then benefitted from attempting to secure a territory by not migrating - something that modelling of the type described below may give an answer to as well. In this paper I will show two ways in which the above stated limitations of theory can be lifted one by one.

\section{Model 1. Body size specific decisions under frequency dependence}

In this section, I follow earlier modelling (in particular Kokko 2007) in that the priori residence effect is assumed absolute. Takeovers of territories are not possible within a year (a bird however has to compete for a new territory after each breeding season). This means that so far I assume that survived non-migrants have better access to high quality habitat than survived migrants. However, I relax the assumption that individuals are all identical, by considering that there is a trait that determines how well a bird is able to survive migration or non-migration. For simplicity throughout this paper I refer to this trait as body size, and assume it is measurable as the bird's weight, which in turn is normally distributed with a mean of $15 \mathrm{~g}$ and standard deviation of one g. Obviously none of the conclusions are dependent on this precise interpretation (or numerical scale) of this trait; it can refer to any property that varies among individuals with predictable effects on survival probability (and later, on RHP). A more detailed examination of strategies could take into account that weight and skeletal size are not perfectly correlated, but as this would require considering faster scale fluctuations in the former than the latter, I leave this for the future.

When individuals differ in their body size, it is often a reasonable assumption that bigger individuals are better off (though see Lind et al. 1999 and Brodin 2007 for avian exceptions). Here I assume that survival increases with weight, but that this relationship can be very different depending on whether the bird migrates or not. Migration requires adaptations for flight, while competition for scarce winter resources might favour large-bodied individuals. Large size could also help maintain body condition during food scarcity that may combine with cold temperatures (Ketterson and Nolan 1976). As an illustrative example I therefore assume that survival increases with body size more strongly in non-migrants than in migrants.

Because competition for territories depends on total population size which again depends on migratory decisions of individuals (Kaitala et al. 1993, Taylor and Norris 2007), it is important to be explicit about population dynamics and hence the distribution of habitat qualities that determine reproductive success. Following Kokko (2007), I assume that territories are finite in number and of variable quality. Ranking the territories in order of declining quality, I assume that the $n$th territory gives rise to, on average, $a-b(n-1)$ female offspring (and equally many males; we concentrate on female population dynamics here because female-to-female reproduction is the prime determinant of whether the population grows or shrinks). Here $a$ indicates the reproductive success that the best territory yields, and $b$ is a small positive constant describing how fast reproductive success declines with territory number. In total there are $[(a+b) / b]-1$ territories suitable for breeding, e.g. $a=2$ and $b=0.001$ yields 2000 territories in which birds can breed. This is obtained by noting that territory number $n=(a+b) / b$ predicts zero success and is thus no longer considered suitable for breeding.

When habitat quality decreases linearly with territory number, a population of non-migrants that have survived the winter will inhabit the first $n_{\mathrm{n}}$ territories $\left(n_{\mathrm{n}}\right.$ denoting the number of such survivors). Their average reproductive 
success, denoted $R_{\mathrm{n}}$, is the average of $a$ and $a-b\left(n_{\mathrm{n}}-1\right)$ (the mean of best and worst territories occupied by nonmigrants), which gives $R_{\mathrm{n}}=a-b\left(n_{\mathrm{n}}-1\right) / 2$. If the number of migrants that have survived is $n_{\mathrm{m}}$, then the average reproductive success of a survived non-migrant is calculated similarly, but since migrants arrive after non-migrants have occupied their preferred territories, the reproductive success of the average migrant is lower:

$$
\begin{aligned}
R_{\mathrm{m}} & =\left[a-b n_{\mathrm{n}}+a-b\left(n_{\mathrm{n}}+n_{\mathrm{m}}-1\right)\right] / 2 \\
& =a-b n_{\mathrm{n}}-b\left(n_{\mathrm{m}}-1\right) / 2 .
\end{aligned}
$$

When larger individuals are relatively better able to cope with the risks of non-migration, and the success of non-migrants as breeders declines with the number of non-migrants in a frequency-dependent fashion $\left(R_{\mathrm{n}}\right.$ declines with $n_{\mathrm{n}}$ as indicated above), there will be a threshold of body size below which a bird is better off migrating than trying to compete for the best territories as a non-migrant. The way to solve for this threshold $S^{*}$ is outlined in the Appendix 1. Briefly, the task is to evaluate the expected reproductive success of migrants and non-migrants $\left(R_{\mathrm{n}}\right.$ and $\left.R_{\mathrm{m}}\right)$ for every possible value of this threshold currently used in the population. This requires that we find the population dynamic equilibrium $\left(n_{\mathrm{n}}\right.$ and $n_{\mathrm{m}}$ ) that is the consequence of a particular value of the size threshold. Then, we disregard all such equilibria as evolutionarily unstable if birds whose size equals the threshold have unequal fitness depending if they migrate or not. If they should clearly migrate, then there are too few migrants in the population for this to be the true frequency-dependent equilibrium, and similarly for the case where non-migration is the better option for them. This leaves just one value which is the evolutionarily stable $S^{*}$.

Unlike many earlier models that assumed no variation in individual traits impacting survival probabilities, this model can produce partial migration based on some individuals surviving better as non-migrants and others as migrants. Figure 3 shows an example where risks experienced as a migrant are less strongly dependent on body size than risks experienced as a non-migrant. Simultaneously, the prior residence effect selects against migration in general. This makes the matter more complicated than stating that individuals choose the option that yields best individual-specific survival. Such a statement would predict, in the example of Fig. 3, that all birds weighing less than $15 \mathrm{~g}$ migrate and all birds above this threshold are year-round residents. It is easy to see why such a supposition is incorrect. At the threshold of $15 \mathrm{~g}$ it does not matter for the survival of the bird whether it migrates or not, but by migrating the birds places itself at a disadvantage when attempting to acquire territories. Birds weighing precisely $15 \mathrm{~g}$ consequently should not migrate, and the migration threshold will consequently evolve towards a lower body size. The method described in the Appendix 1 gives the correct
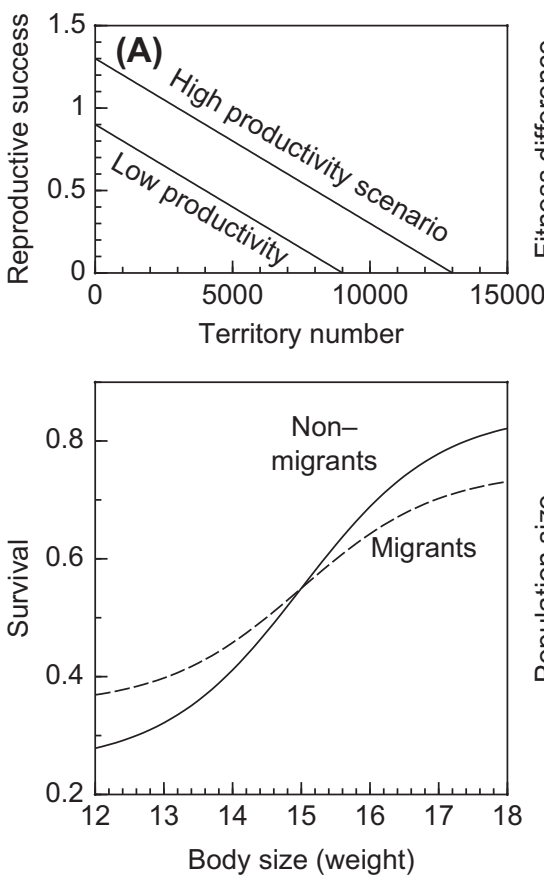
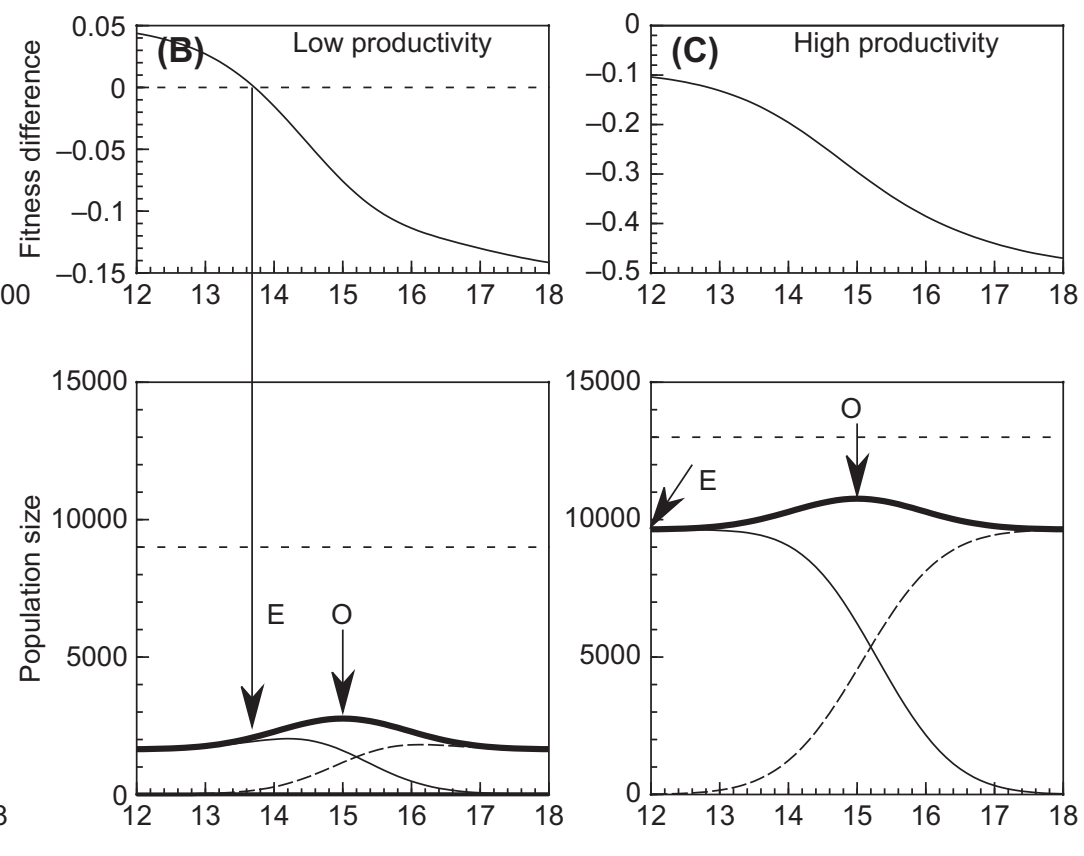

Migration threshold $S$

Figure 3. (A), model assumptions for Model 1. Upper row: the relationship between territory number and its productivity for two different scenarios (upper line: $a=1.3, b=0.0001$, lower line: $a=0.9, b=0.0001)$. Lower row: the assumed survival functions for different body sizes if the bird migrates (dashed line: survival $\left.=0.35+0.4 /\left(1+e^{-(s-15)}\right)\right)$ or not ( solid line: survival $\left.=0.25+0.6 /\left(1+e^{-(s-15)}\right)\right)$. $(\mathrm{B})$, solutions for the low productivity scenario $(a=0.9)$. Upper row: fitness difference between migrating and non-migrating options for birds whose body size equals the currently used migration threshold $S$. This difference declines in a frequency-dependent fashion (at larger $S$, more birds migrate, and the relative benefit of migration diminishes). It reaches zero at $S=S^{*}=13.7 \mathrm{~g}$. Lower row: the number of territories (horizontal dashed line), and the spring population size that evolves to use it (dashed: migrants, solid: non-migrants, heavy solid: total). This size depends on the migration threshold $S$ in use. Evolved behaviour $S^{*}$ leads to a lower population size (arrow E) than if populations optimized survival (arrow O). (C), As (B), but productivity is now higher $(a=1.3)$, and no bird of any size now benefits from migrating even though all birds below $15 \mathrm{~g}$ would have higher survival doing so. Again, evolved population sizes are lower (arrow E) than what would be possible if populations optimized survival (arrow O). 
behavioural rules, which for the examples in Fig. 3B-C are 'migrate if below $13.7 \mathrm{~g}$, and 'never migrate', respectively.

The only difference between Fig. 3B and $3 \mathrm{C}$ is that territories are assumed to be more productive in Fig. 3C. This intensifies competition for breeding sites: there is more total habitat available in Fig. 3C than 3B, but a much larger fraction of it is occupied at equilibrium because the total population evolves to be larger (a population dynamic result of highly productive territories). Intense competition makes year-round residency more important as latecomers are only allowed access to very poor territories. This is a clear demonstration that survival differences between migrants and non-migrants are not sufficient to predict the evolved pattern (determinants of survival are identical across Fig. 3B and 3C). One must consider the population consequences - especially population size - of behaviour to predict the success of each strategy.

It is interesting to note that this model repeats the finding outlined in the introduction, that individuals accept higher risks as non-migrants and thus population size can decline due to competitive site tenacity on the breeding grounds. This happens both in the case of partial migration (Fig. 3B, where lightweight birds migrate) and when the population entirely abandons migration (Fig. 3C). In the former case, our example predicts a breeding population size of 2076 individuals (arrow 'E' in Fig. 3B), out of a maximum of 2758 (arrow 'O') which could be reached if birds simply chose behavioural options to maximize their survival chances in the non-breeding season. Roughly $20 \%$ of the habitat's carrying capacity is 'wasted' on intraspecific competition. In the latter case (Fig. 3C), the corresponding numbers are 9646 out of a maximum of 10758 , i.e. $10 \%$ 'wastage'.

\section{Model 2. Towards more realism: takeovers can occur and individuals follow rules of thumb}

The above model makes use of a number of simplifications that one could conceivably criticize. Most importantly, the simplified method of calculating fitness at the migration threshold implicitly assumes that body sizes are distributed anew each year among birds. In reality, there are likely to be temporal correlations extending to intergenerational effects. Also, the equations provided only work in their current form if territory qualities follow a particular distribution depicted in Fig. 3A. However, instead of providing the next steps to overcome features judged unrealistic, I consider the message of Model 1 to be best complemented by proceeding to flesh out an entirely different method that can deal with complexities more flexibly than any method that attempts to derive very precise behavioural rules (reaction norms of the type 'always migrate if below or above a particular threshold, never migrate otherwise').

The rationale for assuming that individuals cannot optimize all migration-related traits independently across all situations follows from data (Pulido et al. 1996, Pulido and Berthold 2010) but also from general reasoning such as the insights recently provided by McNamara and Houston (2009). These authors argue that it is often more fruitful to look for relatively simple rules that work well under a wide range of scenarios, than to expect very precise optimization for every possible situation (say, body size relative to competitors) that an individual may find itself in. Partial migration is a very clear example where such thinking applies, especially when we consider the complexities of survival together with the sequential nature of the territory establishment phase. Survival, the ability to resist takeovers, or the success rate of an usurper can all depend on body size or related traits in potentially complicated ways, and it is more reasonable to assume that selection has worked out well working rules of thumb (e.g. an increasing or decreasing probability of migrating depending on body size) than precisely optimal behaviour for every microgram of weight difference. The task is then to ask which rules of thumb will be selected for in an environment created by the mix of the rules of thumb currently in use by the population.

I consider this problem using an individual-based simulation model where migration and non-migration decisions follow probabilistically from a reaction norm that relates body size (again taken to be synonymous with weight) to the probability of migration. This reaction norm is assumed to take the shape of the logistic function: the probability of migration $=\left(1+e^{-p_{1}\left(s-p_{2}\right)}\right)^{-1}$, where $p_{1}$ and $p_{2}$ are parameters that dictate the shape of the reaction norm and are allowed to evolve, and $s$ is the bird's size, now used as a proxy for its resource holding power, RHP. The logistic shape is chosen for two reasons. Firstly it is a simple and suitably shaped function (e.g. bounded, such that probabilities do not exceed the $0-1$ range) with enough flexibility to yield e.g. virtually unconditional migration or non-migration. Secondly, it is linked to a statistical technique (logistic regression) which is of the type a researcher is likely to use when portraying how binary decisions - migrate or not - relate to a continuous character. Indeed I will report the outcome of the simulation using logistic regression on what an outside observer, who does not know the program used to generate data, would find in a single generation of the population after evolution has taken place. I adopt this approach to make the output as similar in its interpretation to natural findings as possible.

The details of the simulation are given in Appendix 2; here I give the main assumptions. Birds are, again, normally distributed in weight ranging from 12 to $18 \mathrm{~g}$, with mean 15 and standard deviation of $1 \mathrm{~g}$. Contrasting with Model 1 , I no longer assume that birds that survived the winter on their breeding grounds have absolute prior access to the habitat. Instead, they fill in this habitat up to their total number, and thereafter migrants fill in the available vacancies. (For simplicity, in this model all habitat is of equal quality but there are only a limited number of possible territories.) The habitat may become fully occupied at either stage. If this happens, then each individual who does not have a territory can challenge up to $n$ randomly chosen territory owners. The winner of the challenge is determined according to the prior residency effect rules depicted in Fig. 2. The strength of the prior residence effect is indicated as the weight difference required for the heavier contestant, in the role of an intruder, to be equally likely to win a fight than a lighter owner. Zero effect means that heavier individuals are always more likely to win fights, and lightweight individuals are unlikely to keep their territories if challenged, while effect $=5$ means that a full $5 \mathrm{~g}$ difference (which is substantial, given the standard deviation is $1 \mathrm{~g}$ ) is required before half the fights are won by the much heavier intruder. Note that the chances 
that an overwintered bird keeps its prior access to a territory depends not only on its RHP (size) relative to others but also on the total number of intrusions, thus again we have a dependence on total population size.

The results can be summarized as follows.

\section{The stronger the prior residency effect, the fewer birds migrate}

Figure 4A-B derive the results of the individual-based simulation after 100 years of evolution in a population that was initiated with randomly chosen reaction norms (details in Appendix 2). For each level of strength of the prior residence effect, the curve gives the logistic regression for the probability of migrating as a function of body size (weight of bird), as measured by an observer who has data on birds migrating or not migrating, and their body sizes, in year 100. Even though each curve is measured only once and is thus subject to stochastic noise, the clarity of the outcome (which also is repeatable across simulations and unchanged after further generations; not shown) indicates that selection very clearly favours different migratory tendencies depending on the strength of the prior residence effect. If this effect is weak, birds evolve to migrate; if it is very strong, migration disappears; in between there is a range of solutions where partial migration evolves. This general pattern holds true for very different relationships between survival and body size (Fig. 4A-B).

This result makes sense because the benefit of year-round residency relies on the prior residence effect. Weakening the importance of prior residency therefore selects for more migration, and Fig. 4A-B shows that the change in the selective environment can be very strong - from never migrating to complete migration without any change in survival or reproductive parameters. One detail may appear counterintuitive about this change. The strongest effects of prior residency lead to no migration of any kind (partial or otherwise) in this model, when other models, including Model 1 (Kokko and Lundberg 2001 and Kokko 2007 provide further examples), have been able to produce partial migration assuming an even stronger effect of prior residence, as in such models no takeover is ever successful. This difference becomes explicable, however, once one notes that in Model 2 latecomers must float and remain without any breeding success in the current season if their takeovers are not successful. In Model 1 they could at least acquire low quality territories to breed in. Model 1 therefore assumes a world that is relatively more forgiving to latecomers while in Model 2 latecomer success totally relies on prospects of takeovers being successful, thus the precise strength of the prior residence effect is crucial in this latter model (and probably in all natural situations where latecomers are likely to end up without a territory i.e. floating - rather than breeding in low quality habitat).

\section{The relationship between body condition and survival determines which birds migrate}

Figure 4A-B both assume that heavy birds are more likely to survive the winter as a non-migrant than light birds. However, Fig. 4A assumes that this survival advantage shows itself during migration too (e.g. heavy individuals might be dominant and secure access to food wherever they are, or they may simply have more resources for long flights), while Fig. $4 \mathrm{~B}$ assumes that migration is equally hazardous to birds of any weight. Consequently, the reaction norms that evolve for partial migrants differ in their direction. In Fig. 4A, heaviest birds are more likely to migrate. This is the essence of the 'tropical kingbird' scenario of Jahn et al. (2010), where a dominant bird can enjoy enhanced survival by migrating and then secure access to high quality habitat by taking over territories of non-migrant birds. Regarding the question of why non-migrants tried year-round residency only to get their territories taken over, it is again instructive to think about population dynamics. Very heavy birds are rare and even if each of them is a successful usurper, most non-migrants may be lucky enough to escape ever meeting such a heavyweight challenger to their ownership. Simultaneously the prior residence effect, when moderately strong (as it is in the region of partial migration in Fig. 4A), protects average birds against the less extremely dominant usurpers. Again, non-migrant birds evolve to sacrifice some of their survival prospects in their gamble to secure a breeding site.

The situation can be reversed by assuming, like in the example of Fig. 3, that survival curves cross: heavy birds now survive better if not migrating, light ones by migrating. The region of partial migration (Fig. 4B) now has reaction norms similar to (but smoother than) Fig. 3, in that lightest birds are more likely to migrate than heavier conspecifics. It may now appear puzzling why heavy birds would ever migrate (they evolve complete migration when the prior residence effect is weak, Fig. 4B). It is likely that these cases of complete migration by the entire population reflect the assumption of Model 2 that an owner who loses its territory is not allowed to immediately become an usurper of another one. In a migratory large population, where prior residency does not confer much of an advantage, the risk of becoming usurped may be so large that it is better to have multiple chances of being an usurper (i.e. be a migrant) than to try to hold on to one's initial ownership (the non-migrant strategy).

\section{Discussion}

The two models of this paper demonstrate how restrictive model assumptions can be lifted to yield better understanding of facultative partial migration in birds. Competition for good habitat can drive much of the population to be sitetenacious year-round even if this is costly, in terms of survival, to individuals. These costs are also visible at the population level. To paraphrase Boyle (2008), individuals who 'gamble successfully on year-round residency' will have higher success. But an essential feature of any gamble is that not all attempts will succeed, and there are some relatively counterintuitive consequences of this phenomenon. Most notably, if the conditions during non-breeding season become easier to deal with - say, because of climate change reducing the severity of the winter - this may select for many more individuals participating in the dangerous gamble of yearround residency. Despite the reduced per capita mortality for non-migrants in such a scenario, the total number of deaths can increase because fewer individuals migrate, which would have been the safest option.

A tacit assumption in discussions of evolution of migration under climate change is that populations can decline if genetic adaptation or phenotypic plasticity do not occur fast 

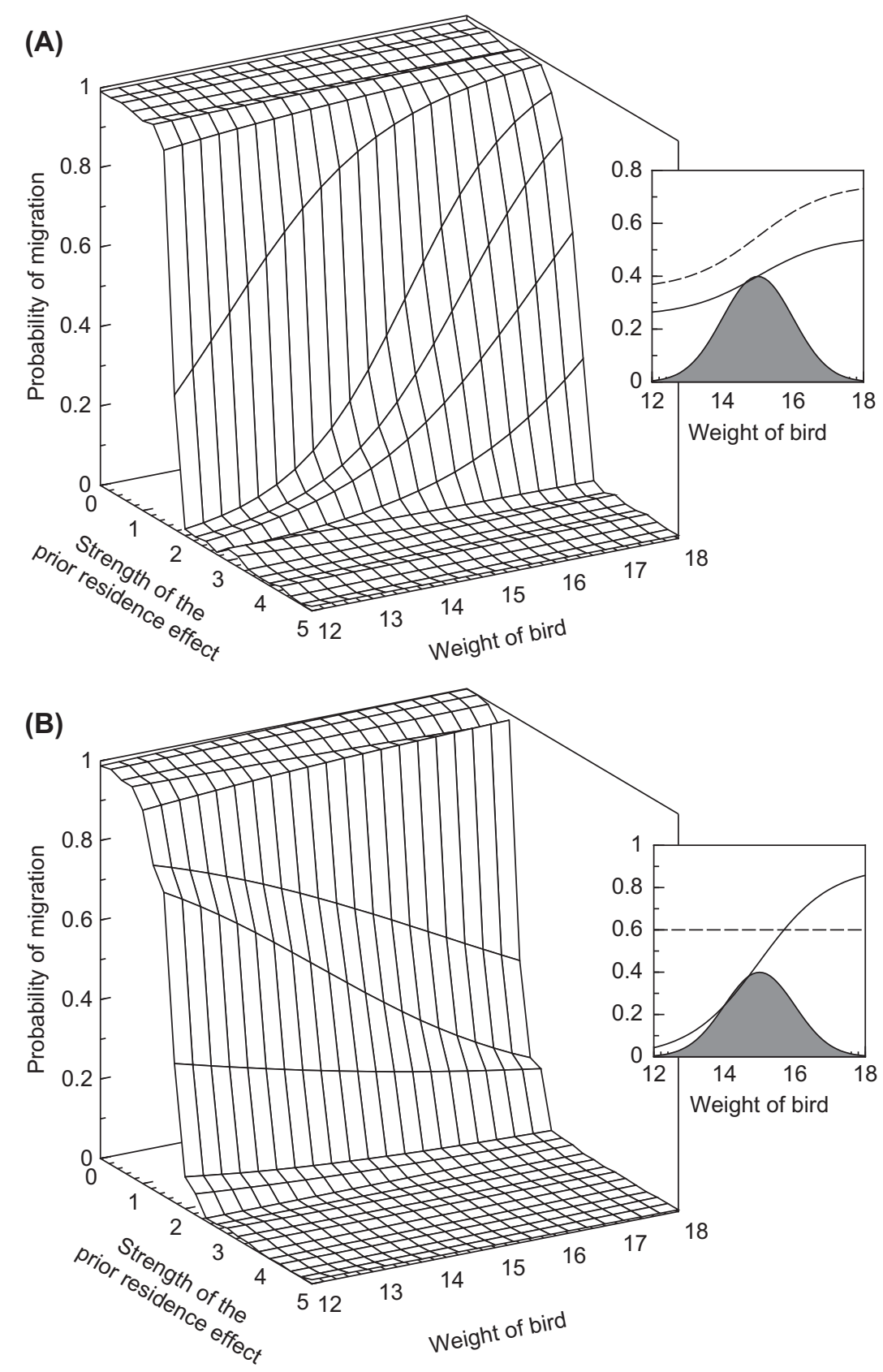

Figure 4. Large figures: a series of logistic regressions, one for each strength of the prior residence effect, performed on a population evolving for 100 years, taking the migratory decision as the dependent variable and body size (weight) as the explanatory variable. Small figures: the corresponding model assumptions regarding survival (dashed: migrants, solid: non-migrants) and weight distribution (filled) that underlie the results. In $(\mathrm{A})$, survival $=0.35+0.4 /\left(1+e^{-(s-15)}\right)$ for migrants and $0.25+0.3 /\left(1+e^{-(s-15)}\right)$ for non-migrants; (B), 0.6 for migrants and $0.9 /\left(1+e^{-(s-15)}\right)$ for non-migrants.

enough (Visser et al. 2004, Both et al. 2006, Charmantier et al. 2008). It is surprising that the opposite - adaptation itself may lead to a population decline - can also be true. This evolutionary prediction, while counterintuitive, is a logical consequence of situations where selection favours individuals that attempt to outcompete conspecifics others in a zerosum fashion (i.e. competitive success comes at the expense of others). When selection makes all individuals invest in competitive traits but resource limitation means that only some will get the reward, population performance will decrease with increasing investment in competitive traits (reviewed by Rankin et al. 2007; see López-Sepulcre et al. 2009 for an empirical example in a non-migratory context). There is evidence that adaptive changes in migratory populations can be quick (Pulido and Berthold 2010, though see also Coppack and Both 2002, Nilsson et al. 2006, Both 2010). If this combines with intensified intraspecific competition, the response of the population can be an equally quick decline. The examples derived here show that these detrimental consequences of competition are not necessarily mild.

Another important result of the current modelling exercise is that slight alterations of the dynamics of territory 
acquisition can lead to very different migratory patterns. Without changing any assumptions regarding survival, and by only changing the relative importance of prior residency vs. resource-holding power in contests over territory ownership, Model 2 is able to predict a switch from complete year-round residency to complete migration. At least one empirical study has already hinted at the dynamics of territory acquisition being crucial to our understanding of partial migration (Jahn et al. 2010), but even this study was not able to document the precise rules that govern this part of the breeding cycle. It follows that much more research effort should be devoted to understanding the dynamics of territory acquisition in partial migrants.

The two models also shed light on the different hypotheses that have attracted specific names when they have been put forward to explain partial migration. There is an interesting cultural difference between empirical and theoretical studies in this context. Empirical studies very often mention, and attempt to distinguish between, specifically named hypotheses (Boyle 2008). The dominance hypothesis states that it would be difficult for a subdominant individual to survive the winter on the breeding grounds, and this predicts year-round residents to be dominant and perhaps large birds. The body-size hypothesis is similar, but the causal route from large size to high survival emphasizes the benefits that a large body confers in harsh and food-limited environments. The arrival time hypothesis emphasizes the importance of acquiring a territory early (for the reproductive prospects), with year-round residency seen as analogous to very early arrival. Finally, seasonal food limitation has been recently presented as a new hypothesis, driven by the fact that not all specific predictions of the above list are met in particular systems (Boyle 2008, Jahn et al. 2010).

The angle taken by most modelling work, however, is to avoid classifying predictions in this way. Instead, models attempt to find out how frequency dependence operates, and guide us to look for mechanisms that make a strategy (say, year-round residency) succeed less well if the proportion of individuals using this strategy increases. The models of the current paper form a case in point. Since the availability of breeding opportunities, via habitat limitation, is the important environmental factor that responds to the behavioural norms used in the population, one could think of these models as belonging to the 'arrival time hypothesis' category. Indeed, costs paid by year-round residents who gamble to get the best reproductive opportunities are very analogous to costs that earliest migrants are predicted to pay in their quest to acquire the best territories (Kokko 1999).

Even so, I believe that a too straightforward association of these models with one hypothesis would mislead. While these models incorporate the importance of securing a breeding site ahead of conspecific competitors, they are simultaneously able to incorporate effects of dominance by adjusting the relative importance of resource holding power (effectively dominance) versus prior residency. Likewise, they can include effects of body size on survival prospects, and these prospects, if necessary, can also be diminished when the aim is to reflect limitations on foraging efficiency. In each case the net effect of all factors determines the migratory outcome. Although the particular models presented here have only made reproduction (not survival) depend on the presence of other, possibly more dominant individuals, other models in turn have modelled survival in a density dependent manner (Kaitala et al. 1993, Taylor and Norris 2007). The general lesson, then, is that partial migration can result from frequency-dependent declines in survival (Kaitala et al. 1993) or reproductive success (Kokko 2007) or both (Griswold et al. 2010) when the number of individuals using the same strategy increases. This shifts emphasis away from distinguishing between models, towards understanding how each effect contributes to the overall pattern.

Although much of behavioural ecology is devoted to distinguishing between clearly outlined hypotheses, one should not succumb to the temptation of putting all research effort into attempts to distinguish between them, with the (usually false) hope that one will end up supported and all others rejected. If the real world typically has different mechanisms acting simultaneously, it may be more fruitful to strive to understand the relative importance of different frequencydependent effects in each system together with the differences in individuals' sensitivity to such effects, than to focus on the most straightforward predictions of each hypothesis if they were acting alone. For instance, in a very impressive study that happens to reject the specific predictions of the dominance hypothesis, Jahn et al. (2010) also suggest that dominance can play a very large role in helping migrants acquire territories. It is somewhat unfortunate, then, that the name 'dominance hypothesis' has been reserved for a very specific effect of dominance (survival in the non-breeding season), which differs from its effect on territory acquisition, argued to be important for the kingbirds studied by Jahn et al. (2010). 'Dominance hypothesis rejected' is therefore not synonymous with 'dominance is not needed to explain the system'.

There is, however, also an upside to exposing the expectations that are based on a single mechanism. This is the ease of relating results to work conducted in a parallel area, that of arrival timing in migrants. A major omission from all theoretical work on partial migration so far, including the models presented in the current paper, is that two sexes are not explicitly considered. However, by viewing the results in the light of a single mechanism - that outlined in the arrival time hypothesis - an analogy can be drawn to two-sex models that predict arrival times for species with complete migration. The models here show that frequency-dependent competition for breeding sites alone can be sufficient to drive partial migration, and thus - in a minor deviation from Taylor and Norris (2007) - survival does not necessarily have to vary with density in either the breeding or non-breeding season for there to be sufficient frequency-dependence in reproductive output that maintains coexisting strategies. The arrival time hypothesis concentrates on this effect on reproductive payoffs, and it is usually interpreted to predict that males should arrive earlier than females (Boyle 2008). By analogy, then, in partial migrants males should migrate less than females. However, this prediction has been shown to be fallible in the context of arrival timing in completely migratory populations (Kokko et al. 2006b), unless adult sex ratios are male-biased. Without such a bias, it is hard to understand why only one sex would benefit from outcompeting same-sex competitors in an attempt to secure a good breeding site, when the reproductive success of both sexes depends on habitat quality and availability. 
Given that adult sex ratios in fact often are male-biased in birds (Donald 2007), the above caveat may not be a serious one for many species, because when higher numbers of males than females compete against same-sex individuals for the same breeding sites, this creates relatively more intense selection on males than on females to remain close to potential breeding sites. This restores the proposed causality from the need to secure best territories to a male-biased occurrence of year-round residency. Still, given that this argument only applies with caveats (Kokko et al. 2006b), and despite much empirical study devoted to considering sex differences (Smith and Nilsson 1987, Boyle 2008), it is notable that no model has explicitly treated territory acquisition and pair formation by the two sexes in partial migrants. A partial exception is provided by Griswold et al. (2010) who consider two sexes, but mating is random in their model and males and females are not permitted to evolve sex-specific expression of migratory behaviour. Clearly, there is much work to do in deriving explicit predictions of the population feedback when the number of males can differ from the number of females, especially because sex-specific habitat use can influence determinants of fitness in the non-breeding season too (Studds and Marra 2005).

Finally, it is worth noting that despite including several interacting factors, the models presented here do not cover all forms that partial migration is known to take in nature. The models are formulated with birds in mind. While the general conclusions regarding the importance of frequency dependence are likely to hold generally, the particular mechanisms can vary from taxon to taxon. Even within birds, partial migration is known to sometimes take the form of all individuals being in the same region for the non-breeding season (Middleton et al. 2006), instead the breeding range being shared among all individuals, as modelled here. The fitness consequences obviously have to be calculated in a different way for the former case (for an explicit comparison see Griswold et al. 2010). Despite limitations, current theory does point out several avenues for fruitful research: a simultaneous evaluation of survival prospects and territory acquisition dynamics is essential to understand frequency dependence; the study of interactions between the sexes is in its infancy; and the study of population consequences of individual migratory decisions can yield surprising results.

Acknowledgements - I wish to thank the organizers of the Partial migration symposium in Lund - particularly Ben Chapman - for providing a friendly and stimulating atmosphere, and the Indian Pacific train service for prolonged views of the Nullarbor and limitless caffeine. Funding was provided by the Australian Research Council.

\section{References}

Both, C. 2010. Flexibility of timing of avian migration to climate change masked by environmental constraints en route. - Curr. Biol. 20: 243-248.

Both, C. et al. 2006. Climate change and population declines in a long-distance migratory bird. - Nature 441: 81-83.

Boyle, W. A. 2008. Partial migration in birds: tests of three hypotheses in a tropical lekking frugivore. - J. Anim. Ecol. 77: 1122-1128.
Boyle, W. A. 2011. Short-distance partial migration of Neotropical birds: a community-level test of the foraging limitation hypothesis. - Oikos 120: 1803-1816.

Boyle, W. A. and Conway, C. J. 2007. Why migrate? A test of the evolutionary precursor hypothesis. - Am. Nat. 169: 344-359.

Brodin, A. 2007. Theoretical models of adaptive energy management in small wintering birds. - Phil. Trans. R. Soc. B 362: 1857-1871.

Chapman, B. B. et al. 2011. The ecology and evolution of partial migration. - Oikos 120: 1764-1775.

Charmantier, A. et al. 2008. Adaptive phenotypic plasticity in response to climate change in a wild bird population. - Science 320: 800-803.

Coppack, T. and Both, C. 2002. Predicting life-cycle adaptation of migratory birds to global climate change. - Ardea 90: 369-378.

Donald, P. F. 2007. Adult sex ratios in wild bird populations. - Ibis 149: 671-692.

Griswold, C. K. et al. 2010. The evolution of migration in a seasonal environment. - Proc. R. Soc. Lond. B 277: 2711-2720.

Hardy, I. C. W. and Field, S. A. 1998. Logistic analysis of animal contests. - Anim. Behav. 56: 787-792.

Jahn, A. E. et al. 2010. Determinants of partial bird migration in the Amazon basin. - J. Anim. Ecol. 79: 983-992.

Kaitala, A. et al. 1993. A theory of partial migration. - Am. Nat. 142: 59-81.

Ketterson, E. D. and Nolan, V. Jr. 1976. Geographic variation and its climatic correlates in sex-ratio of eastern wintering dark-eyed juncos (Junco hyemalis hyemalis). - Ecology 57: 679-693.

Kokko, H. 1999. Competition for early arrival in migratory birds. - J. Anim. Ecol. 68: 940-950.

Kokko, H. 2007. Modelling for field biologists and other interesting people. - Cambridge Univ. Press.

Kokko, H. and Lundberg, P. 2001. Dispersal, migration and offspring retention in saturated habitats. - Am. Nat. 157: $188-202$.

Kokko, H. et al. 2006a. From hawks and doves to self-consistent games of territorial behavior. - Am. Nat. 167: 901-912.

Kokko, H. et al. 2006b. Why do female migratory birds arrive later than males? - J. Anim. Ecol. 75: 1293-1303.

Lind, J. et al. 1999. Reduced take-off ability in robins (Erithacus rubecula) due to migratory fuel load. - Behav. Ecol. Sociobiol. 46: 65-70.

López-Sepulcre, A. et al. 2009. Reproductive conflict delays the recovery of an endangered social species. - J. Anim. Ecol. 78: 219-225.

Lundberg, P. 1987. Partial bird migration and evolutionarily stable strategies. - J. Theor. Biol. 125: 351-340.

Lundberg, P. 1988. The evolution of partial migration in birds. Trends Ecol. Evol. 3: 172-175.

McNamara, J. M. and Houston, A. I. 2009. Integrating function and mechanism. - Trends Ecol. Evol. 24: 670-675.

Middleton, H. A. et al. 2006. Breeding territory fidelity in a partial migrant, the American dipper Cinclus mexicanus. - J. Avian Biol. 37: 169-178.

Nilsson, A. L. K. et al. 2006. The effect of climate change on partial migration - the blue tit paradox. - Global Change Biol. 12: 2014-2022.

Pulido, F. and Berthold, P. 2010. Current selection for lower migratory activity will drive the evolution of residency in a migratory bird population. - Proc. Natl Acad. Sci. USA 107: 7341-7346.

Pulido, F. et al. 1996. Frequency of migrants and migratory activity are genetically correlated in a bird population: evolutionary implications. - Proc. Natl Acad. Sci. USA 93: 14642-14647.

Rankin, D. J. et al. 2007. The tragedy of the commons in evolutionary biology. - Trends Ecol. Evol. 22: 643-651.

Sandell, M. and Smith, H. G. 1991. Dominance, prior occupancy, and winter residency in the great tit (Parus major). - Behav. Ecol. Sociobiol. 29: 147-152. 
Sinervo, B. and Calsbeek, R. 2006. The developmental, physiological, neural and genetical causes and consequences of frequency-dependent selection in the wild. - Annu. Rev. Ecol. Evol. Syst. 37: 581-610.

Smith, H. G. and Nilsson, J. A. 1987. Intraspecific variation in migratory pattern of a partial migrant, the blue tit (Parus caeruleus): an evaluation of different hypotheses. - Auk 104: 109-115.
Studds, C. E. and Marra, P. P. 2005. Nonbreeding habitat occupancy and population processes: an upgrade experiment with a migratory bird. - Ecology 86: 2380-2385.

Taylor, C. M. and Norris, D. R. 2007. Predicting conditions for migration: effects of density dependence and habitat quality. Biol. Lett. 3: 280-283.

Visser, M. E. et al. 2004. Global climate change leads to mistimed avian reproduction. - Adv. Ecol. Res. 35: 89-110. 


\section{Appendix 1}

\section{Deriving the evolutionarily stable size threshold in Model 1}

The first step in finding the evolutionarily stable size threshold $S^{*}$ is to derive the population consequences (most importantly, $R_{\mathrm{m}}$ and $R_{\mathrm{n}}$ ) of any threshold $S$ currently used in the population. We are also interested in the spring population size of migrants and non-migrants, $n_{\mathrm{m}}$ and $n_{\mathrm{n}}$, that describe the population dynamic equilibrium. The spring population (total size $n_{\mathrm{n}}+n_{\mathrm{m}}$ ) yields on average, per breeder, $a-\left(b\left(n_{\mathrm{n}}+n_{\mathrm{m}}-1\right) / 2\right)$ new members to the population by the autumn, and the breeders $\left(n_{\mathrm{n}}+n_{\mathrm{m}}\right)$ are still alive too. All these birds follow the normal distribution for body weight. Denoting the probability density function for size $s$ by $f(s)$, the total number of birds of size $s$ in the autumn is $\left(n_{\mathrm{n}}+n_{\mathrm{m}}\right)$ $\left[1+a-\left(b\left(n_{\mathrm{n}}+n_{\mathrm{m}}-1\right) / 2\right)\right] f(s)$. If this size $s$ falls below the migration threshold $S$, then size $s$ birds all migrate, and the total number of birds of size $s$ that return alive the following spring is

$\left(n_{\mathrm{n}}+n_{\mathrm{m}}\right)\left[1+a-\left(b\left(n_{\mathrm{n}}+n_{\mathrm{m}}-1\right) / 2\right)\right] f(s) p_{\mathrm{m}}(s)$

where $p_{\mathrm{m}}(s)$ is the probability of surviving as a migrant at size $s$. By contrast, if the size $s$ exceeds $S$, then size $s$ birds do not migrate, and the total number of birds of size $s$ that overwinter successfully on their breeding grounds is

$\left(n_{\mathrm{n}}+n_{\mathrm{m}}\right)\left[1+a-\left(b\left(n_{\mathrm{n}}+n_{\mathrm{m}}-1\right) / 2\right)\right] f(s) p_{\mathrm{n}}(s)$,

where $p_{\mathrm{n}}(s)$ is the probability of surviving as a non-migrant at size $s$.

At equilibrium, the number of migrants and non-migrants stays constant from one year to the next: the dynamic equilibrium must satisfy the set of equations

$$
\begin{aligned}
& n_{\mathrm{m}}=\left(n_{\mathrm{n}}+n_{\mathrm{m}}\right)\left[1+a-\left(\frac{b\left(n_{\mathrm{n}}+n_{\mathrm{m}}-1\right)}{2}\right)\right] \int_{-\infty}^{s} f(s) p_{\mathrm{m}}(s) \mathrm{d} s \\
& n_{\mathrm{n}}=\left(n_{\mathrm{n}}+n_{\mathrm{m}}\right)\left[1+a-\left(\frac{b\left(n_{\mathrm{n}}+n_{\mathrm{m}}-1\right)}{2}\right)\right] \int_{S}^{\infty} f(s) p_{\mathrm{n}}(s) \mathrm{d} s
\end{aligned}
$$

The solutions are

$$
\begin{aligned}
& n_{\mathrm{m}}=A \int_{-\infty}^{S} f(s) p_{\mathrm{m}}(s) \mathrm{d} s \\
& n_{\mathrm{n}}=A \int_{S}^{\infty} f(s) p_{\mathrm{n}}(s) \mathrm{d} s
\end{aligned}
$$

where

$$
A=\frac{\left(\int_{-\infty}^{s} f(s) p_{\mathrm{m}}(s) \mathrm{d} s+\int_{s}^{\infty} f(s) p_{\mathrm{n}}(s) \mathrm{d} s\right)(2+2 a+b)-2}{b\left(\int_{-\infty}^{s} f(s) p_{\mathrm{m}}(s) \mathrm{d} s+\int_{s}^{\infty} f(s) p_{\mathrm{n}}(s) \mathrm{d} s\right)^{2}}
$$

gives the closed form solution for the autumn population size.

Although complicated, these solutions are all in closed form, which means that they can be numerically evaluated with ease.
The evolutionarily stable threshold $S^{*}$ can be found using several different methods; for a general pairwise invasibility analysis see Kokko (2007). A shortcut can be found by noting that if at the current size threshold $S$ individuals do better by migrating than by non-migrating, then they should switch to a migrating strategy (and vice versa). If $S$ is evolutionarily stable (such that $S=S^{*}$ ), then at this size $s=S$ individuals should have equal fitness using either behavioural strategy. This implies $p_{\mathrm{m}}(s)\left(R_{\mathrm{m}}+1\right)=p_{\mathrm{n}}(s)\left(R_{\mathrm{n}}+1\right)$, where $R_{\mathrm{m}}$ and $R_{\mathrm{n}}$ are obtained using the equilibrium values of $n_{\mathrm{m}}$ and $n_{\mathrm{n}}$, as described in the main text.

\section{Appendix 2}

\section{The details of the simulation (Model 2)}

A population of 1000 individuals was initiated in a habitat comprising 1000 territories, such that each individual was characterized by three traits: the parameter $p_{1}$ (initially normally distributed with mean 1 and standard deviation 0.1 , but with probability 0.5 its sign was changed to become negative), the parameter $p_{2}$ (initially normally distributed with mean 15 and standard deviation 0.1 , but with probability 0.5 its sign was changed to become negative), and the individual's size $s$ which is interpreted as its weight (normally distributed with mean 15 and standard deviation 1). The combination of signs and magnitudes of $p_{1}$ and $p_{2}$ ensures that the population starts as a mix of individuals whose probability of migration is low or high, and increases or decreases (or remains largely unchanged) with body size. Further variation was then introduced by mutation as described below.

For 100 breeding seasons, the following procedure was followed:

1. Initially the number of birds and territories was matched, such that all 1000 birds are breeders.

2. Each breeder produces $R$ offspring (in the examples shown, $R=3$ ), and these inherit the traits $p_{1}$ and $p_{2}$ from the parent bird but with mutation: for $i=1$ or 2 , the offspring's $p_{\mathrm{i}}$ equals the parent's $p_{\mathrm{i}}$ plus a normally distributed mutation term with mean 0 and standard deviation 0.1 . This type of mutation captures the idea of a polygenic trait (many loci each with small effects). Offspring size is then randomly determined: as with the parents, this is normally distributed with mean 15 and standard deviation 1 . There is no parent-offspring correlation in this trait.

3. Each bird (parent, offspring, or floater) makes its decision to migrate or not. Note that floaters do not exist in the first year but they can arise later. The probability that a bird of size $s$ migrates is $\left(1+e^{-p_{1}\left(s-p_{2}\right)}\right)^{-1}$. If a uniformly distributed random number (in the range 0 to 1 ) falls below this probability then the bird migrates, otherwise it does not.

4. Migrants who died (each which probability given in the legend of Fig. 4) are removed from the population, ditto for non-migrants.

5. We now have the spring population. If the number of survived non-migrants falls below the number of territories (1000), all non-migrants are assigned the 
status of a territory owner. If their number exceeds 1000, then 1000 non-migrants are randomly chosen as territory owners, and the rest are then individually picked in random order to challenge up to five different owners. The fights end for each challenger if it wins a fight (probability determined depending on the strength of the prior residence effect and on the body size of the challenger and owner using the function depicted in Fig. 2), in which case it becomes the new owner and the previous owner is ousted and cannot breed in this season, or if it has challenged five owners without success, in which case the challenger cannot breed this season.

6. The migrants arrive. If the number of territory owners is below 1000 at this stage, the remaining vacancies are filled by migrants. If not all migrants find a territory by this means, then they can challenge current territory owners in the same manner as non-migrants described above. Note that a migrant can challenge either a nonmigrant or a migrant (who obtained a territory earlier in the sequence than the challenger migrant).

7. We have now the new spring population of breeders (territory owners) and, potentially, floaters (birds alive but without a territory). Floaters will simply survive to form part of the autumn population, breeders breed as described from stage 2 onwards.

8. This cycle is repeated until a predetermined number of seasons has passed.

9. A logistic regression is performed on the migration decisions (step 3) of all birds alive in the last breeding season, with the binary outcome - to migrate or not regressed against body size. 